Revista de Economia Política, vol. 20, $n^{\circ} 2$ (78), pp. 312-334, abril-junho/2000

\title{
Valores-Trabalho e Preços de Produção em um Sistema Econômico Sraffiano com Renda Extensiva
}

\author{
Labor Values and Production Prices in a Sraffian \\ Economic System with Extensive Income
}

MARINA SILVA DA CUNHA*

RODOLFO HOFFMANN**

\begin{abstract}
RESUMO: Este artigo desenvolve uma extensão da abordagem de Sraffian, considerando um modelo em que a economia é dividida em dois setores, o industrial e o agrícola. A partir de um sistema em unidades físicas, é possível determinar valores de mão de obra e preços de produção. $\mathrm{O}$ artigo analisa a determinação dos valores trabalhistas usando a sugestão de Morishima, com programação linear. Demonstra-se que em um sistema econômico com escassez de terras a existência de mais-valia positiva não é uma condição suficiente para lucros positivos.
\end{abstract}

PALAVRAS-CHAVE: Análise sraffiana; valor trabalho; renda da terra.

ABSTRACT: This article develops an extension of Sraffian's approach, considering a model where the economy is divided into two sectors, the industrial and the agricultural. From a system in physical units it is possible to determine labour values and prices of production. The paper analyses the determination of labour values using Morishima's suggestion, with linear programming. It is shown that in an economic system with land scarcity the existence of positive surplus-value is not a sufficient condition for positive profits.

KEYWORDS: Sraffian analysis; labor value; property income.

JEL Classification; B51; D46.

\section{INTRODUÇÃO}

O principal trabalho de Piero Sraffa, intitulado Produção de Mercadorias por meio de Mercadorias, foi publicado em 1960. Segundo Steedman (1977), a preocupação central desse livro são as relações entre salários, lucros e preços, para

\footnotetext{
* Professora da Universidade Estadual de Maringá, Maringá/PR, Brasil. E-mail: mscunha@uem.br.

** Professor do Instituto de Economia da UNICAMP e da ESALQ/USP, Piracicaba/SP, Brasil. E-mail: hoffmanr@usp.br.
} 
dadas condições de produção, supondo uniformes em toda a economia o salário, a taxa de lucro e o preço de cada mercadoria.

Este trabalho teve repercussões tanto na escola marginalista quanto na marxista. A partir dessa publicação desenvolveu-se uma crítica formal à teoria marginalista, como indicava o subtítulo do livro: Prelúdio a uma Crítica da Teoria Econômica. A polêmica resultante foi denominada por Harcourt (1972) de Controvérsias de Cambridge sobre a Teoria do Capital e discutiu vários aspectos da metodologia neoclássica, tais como a distribuição de renda, a mensuração do capital e a escolha de técnicas ${ }^{1}$.

$\mathrm{Na}$ escola marxista surgiram trabalhos utilizando modelos sraffianos visando a uma abordagem formal do problema marxista da transformação dos valores em preços. Tais trabalhos empregam modelos que podem ser denominados sraffiano marxistas. Ao contrário do que é feito no esquema original de Sraffa, nos modelos sraffiano-marxistas o custo do pagamento da mão-de-obra (o capital variável de Marx) é incluído no montante sobre o qual se calculam os lucros. Segundo Médio (1973), essa abordagem tornou possível uma prova formal da consistência da teoria do valor de Marx.

Para Roncaglia (1978), o livro de Sraffa representa um retorno às ideias dos economistas clássicos. Um exemplo da retomada do pensamento clássico em Sraffa é sua análise da utilização da terra na produção de mercadorias, uma vez que pode ser considerada uma formulação matemática das ideias de Ricardo (Gibson \& Mcleud, 1983). Segundo Ricardo, Sraffa analisa dois casos: o primeiro em que existem várias qualidades de terra, o que dá origem à renda extensiva, e o segundo em que existem vários métodos ou técnicas de produção empregadas em uma terra homogênea, implicando uma renda de caráter intensivo.

Nesse sentido, a terra pode ser analisada em um modelo com produção simples com processos ou técnicas alternativas para a produção da mercadoria agrícola, isto é, um modelo em que cada processo ou técnica produz apenas uma mercadoria, e a mercadoria agrícola pode ser produzida por mais de um processo ou técnica. Existe uma analogia entre a questão da escolha da técnica e os modelos com a terra como insumo. Como veremos, nos modelos que incluem o setor agrícola, a determinação da margem (extensiva ou intensiva) é uma maneira de abordar a questão da escolha da técnica (Woods, 1990).

$\mathrm{Na}$ abordagem sraffiana, processos de produção que utilizam a terra, ou seja, um meio de produção não produzido, têm sido analisados como o inverso dos processos com produtos conjuntos, isto é, aqueles bens que não podem ser produzidos separadamente, como couro e carne bovina. Esses podem ser vistos como um único processo de produção produzindo vários produtos, enquanto o primeiro tem

\footnotetext{
${ }^{1}$ Segundo Harcourt (1972), o início desse debate foi o artigo de 1953 escrito por Joan Robinson, intitulado "The Production Function and the Theory of Capital" [uma reprodução do mesmo pode ser encontrada em Harcourt \& Lang (1971)], inspirado no prefácio escrito por Sraffa para a edição das Obras de Ricardo, originalmente publicada em 1950. Ver Sraffa, 1982.
} 
sido examinado como o caso de vários processos de produção (terras de diferentes qualidades ou várias técnicas de cultivo em uma terra de qualidade homogênea) que produzem um único produto (Pasinetti, 1980).

A análise de processos com técnicas alternativas na teoria do valor-trabalho ocorreu com a discussão da produção conjunta. Morishima (1973, 1974 e 1976) analisou esses temas segundo uma nova definição de valor-trabalho, os valores verdadeiros, dados pela solução de um problema de programação linear de minimização do tempo de trabalho necessário para obter determinado produto líquido. Steedman (1975 e 1976) formulou um exemplo numérico em termos dos valores efetivos, em que verifica que na presença de produção conjunta e técnicas alternativas podem coexistir uma taxa de mais-valia negativa com uma taxa de lucro positiva. Os valores efetivos de Steedman são aditivos, mas podem ser negativos. Por outro lado, os valores verdadeiros de Morishima nunca são negativos, mas não são aditivos.

Após a publicação de "Produção...", vários autores passaram a estudar e a desenvolver o que Sraffa tinha escrito sobre sistemas de produção com a terra como insumo. Contudo, o setor agrícola ainda não foi analisado nos modelos sraffiano marxistas. Com isso, o principal objetivo do presente trabalho constitui-se em analisar e desenvolver sistemas econômicos sraffiano-marxistas que incluam o setor agrícola, considerando tanto a determinação dos preços de produção quanto a dos valores-trabalho e a produção do cereal em diferentes qualidades de terra.

Além dessa introdução, este trabalho consta ainda de quatro seções. A segunda analisa a determinação dos valores-trabalho em um modelo com o setor industrial e o agrícola e a terceira trata dos preços de produção nesse modelo. A quarta seção apresenta um exemplo numérico hipotético a fim de ressaltar alguns aspectos dos sistemas sraffiano-marxistas desenvolvidos. Por fim, na última seção, são resumidas as conclusões obtidas.

\section{A DETERMINAÇÃO DOS VALORES-TRABALHO²}

Vamos considerar que a economia está dividida em dois setores: o industrial e $\mathrm{o}$ agrícola. $\mathrm{O}$ setor industrial produz $\mathrm{n}_{\mathrm{a}}$ mercadorias, com ausência de técnicas alternativas e produção conjunta, ou seja, cada mercadoria é produzida por uma única técnica e essa produz apenas uma mercadoria. Todas as mercadorias do sistema são básicas. ${ }^{3} \mathrm{Na}$ agricultura, uma mercadoria homogênea, denominada cereal,

\footnotetext{
${ }^{2}$ No trabalho de tese foram analisados outros dois métodos de determinação dos valores-trabalho, o primeiro utilizando o conceito de valores médios e o segundo, o conceito de valores marginais de Fine (1979). Contudo, a abordagem que segue a proposta de Morishima foi a mais consistente com a teoria marxista.

${ }^{3}$ Sraffa (1985) denomina de básica uma mercadoria que entra na produção de todas as mercadorias, direta ou indretamente. Caso contrário, a mercadoria é chamada não-básica.
} 
é produzida em $\mathrm{n}_{\mathrm{z}}$ diferentes qualidades de terra. Assim, a produção da mercadoria agrícola admite técnicas alternativas, ou seja, existe uma técnica específica associada a cada qualidade de terra $m\left(\operatorname{com} m=1, \ldots, \mathrm{n}_{\mathrm{z}}\right)$. Há $n_{a}+n_{z}$ atividades: $n_{a}$ indústrias e $n_{z}$ maneiras de produzir a mercadoria agrícola.

São feitas as seguintes suposições: (i) a economia se encontra em estado estacionário, ou seja, a cada ano produz-se a mesma quantidade física de mercadorias; (ii) todos os meios de produção necessários para a produção de cada mercadoria são consumidos durante o período de produção e devem ser inteiramente repostos, ou seja, todo capital é capital circulante; (iii) todas as técnicas apresentam o mesmo período de produção; (iv) todo excedente é distribuído na forma de salários, lucros e, eventualmente, renda da terra; (v) a força de trabalho é homogênea; (vi) há retornos constantes à escala. ${ }^{4}$

Sejam A e $\mathbf{B}$ matrizes retangulares $\left(n_{a}+1\right) \mathrm{x}\left(n_{a}+n_{z}\right)$, com elementos $a_{i j}$ e $b_{b j}$ $\left(i\right.$ ou $h=1, \ldots, n_{a}+1$ e $\left.j=1, \ldots, n_{a}+n_{z}\right)$, respectivamente. O elemento $a_{i j}$ indica a quantidade da mercadoria $i$ que entra como insumo na produção do conjunto de mercados indicado na $j$-ésima coluna de $\mathbf{B}$ (quantidades $b_{b j} \operatorname{com} b=1, \ldots, n_{a}+1$ ). As quantidades de trabalho empregadas nas $n_{a}$ e $n_{z}$, atividades estão no vetor-linha $\mathrm{b}$.

Adaptando a proposta de Morishima (1974 e 1976) ao caso de um sistema com setor agrícola, o valor-trabalho em um modelo que inclui uma mercadoria agrícola pode ser mensurado em termos dos valores verdadeiros. O valor-trabalho de qualquer cesta de mercadorias (ou de uma mercadoria) é obtido ao resolver um problema de programação linear. Essa cesta de mercadorias é representada por um vetorcoluna y com $n_{a}+1$ elementos. Seja $s$ um vetor-coluna com $n_{z}$, elementos $\left(\mathrm{s}_{\mathrm{m}}\right.$, com $m=1, \ldots, n_{z}$ ), que são as quantidades disponíveis de cada tipo de terra. Indica-se por q um vetor-coluna com $n_{a}+n_{z}$ elementos que são os multiplicadores das atividades, de maneira que $\mathrm{Bq}$ seja o vetor-coluna das quantidades totais produzidas e Aq o vetor-coluna das quantidades de cada mercadoria usadas como insumos. Os últimos $n_{z}$ elementos de $\mathbf{q}$ constituem o vetor-coluna $\mathrm{q}_{\mathrm{z}}$. O produto líquido é $\mathrm{Bq}$ - Aq. Seja $\mathrm{H}$ uma matriz diagonal, $n_{z}, x n_{z}$, onde os elementos da diagonal principal representam as áreas das diferentes qualidades de terra $\left(h_{m}, \operatorname{com} m=1, \ldots, n_{z}\right)$, em hectares, utilizadas para produzir os volumes da mercadoria agrícola dados em B. Com isso, o valor verdadeiro da cesta de mercadorias $\left(v_{y}\right)$ da cesta de mercadorias y é obtido com a resolução do seguinte problema de programação linear:

Minimizar vy $=$ bq
com
$\mathrm{Bq} \geq \mathrm{Aq}+$ y ou $(\mathrm{B}-\mathrm{A}) \mathrm{q} \geq \mathrm{y}$
$\mathrm{Hq}_{z} \leq \mathrm{s}$
$\mathrm{q} \geq 0$

\footnotetext{
${ }^{4}$ Para Garegnani (1977), a suposição de um salário uniforme é uma consequência de que, em todas as indústrias, os salários sejam formados por uma mesma "mercadoria salário composta", à qual pode ser atribuída um mesmo preço resultante dos preços das mercadorias que a compõem.
} 
A equação (2) indica que o produto total de cada mercadoria básica no sistema tem de ser, no mínimo, igual ao que é gasto dessas mercadorias como insumo mais a cesta de mercadorias ou mercadoria composta, cujo valor-trabalho está sendo determinado. A segunda restrição, equação (3), está relacionada ao fato de que a área de cada qualidade de terra cultivada para produzir o cereal não pode ultrapassar o total dessa mesma qualidade disponível no sistema econômico. Por fim, a equação (4) implica que a solução do sistema possui significado econômico, pois não existe produção negativa.

\section{Os valores individuais}

Para Morishima \& Catephores (1980), os valores individuais das mercadorias podem ser obtidos de duas formas. Primeiro, o valor verdadeiro de uma mercadoria $i$ é definido como o valor verdadeiro da mercadoria composta que contém apenas uma unidade da mercadoria $i$, sendo todos os outros elementos no vetor $\mathbf{y}$ iguais a zero. Assim, para obter os valores individuais, apenas o vetor y é modificado, na equação (2). Indicando esses valores individuais por $v_{\mathrm{y}}$ e sendo $y_{i}$ os elementos de y no problema (1)-(4), Morishima \& Catephores (1980) assinalam que:

$$
v_{y} \leq \sum v_{y i} y_{i}
$$

Essa desigualdade indica que a soma dos valores verdadeiros individuais das mercadorias multiplicados por suas respectivas quantidades contidas no vetor y pode ser maior do que o valor verdadeiro de y. Em outros termos, “(...) o valor verdadeiro de uma mercadoria composta não é aditivo em relação a seus componentes, exceto em alguns casos e speciais"5 (Morishima \& Catephores, 1980, p. 45).

Veremos que com o setor agrícola podemos obter, também:

$$
v_{y}>\sum v_{y i} y_{i}
$$

contrariando a equação (2.3) de Morishima \& Catephores (1980).

Segundo, podemos relacionar os valores verdadeiros ao que Morishima (1974) denominou de valores ótimos. Os valores ótimos $\left(v_{o}\right.$ são as variáveis de escolha de um problema de maximização que é dual do problema de minimização. Para encontrar o problema dual descrito nas equações (1) - (4), primeiro devemos multiplicar a restrição (3) por -1 , para que todas as desigualdades do problema de minimização fiquem com o mesmo sentido. Posteriormente, para cada linha e coluna desse problema de minimização devemos obter, correspondentemente, uma coluna e uma linha no problema dual. Seja $\mathrm{v}_{t}$ um vetor-linha $\operatorname{com} n_{z}$ elementos, que são os valores-sombra dos vários tipos de terra, e seja $\mathrm{v}_{o}$ um vetor-linha com $n_{a}+1$ elementos com os valores-trabalho ótimos das mercadorias industriais e do cereal.

\footnotetext{
${ }^{5}$ Esses casos especiais correspondem aos sistemas sem produção conjunta e sem técnicas alternativas.
} 
Então, os valores-trabalho ótimos em um sistema com uma mercadoria agrícola podem ser obtidos com a resolução do seguinte problema de programação linear:

$$
\text { Maximizar } \mathrm{Z}=\left[v_{0}-v_{t}\right]_{\mathrm{s}}^{\mathrm{y}}
$$

com

$$
\begin{aligned}
& \mathrm{v}_{0}(\mathrm{~B}-\mathrm{A})-\left[0 \mathrm{v}_{\mathrm{t}} \mathrm{H}\right] \leq \mathrm{b} \\
& \mathrm{v}_{0} \geq 0 \mathrm{c} \mathrm{v}_{t} \geq 0
\end{aligned}
$$

$\mathrm{Na}$ expressão (8), o 0 representa um vetor-linha com $n_{a}$ elementos iguais a zero.

Se $Z^{0}$ é a solução do problema de maximização e bq ${ }^{0}$, na equação (1), a solução do problema de minimização correspondente, então pelo teorema da dualidade teremos ${ }^{6}$

$$
\begin{aligned}
& \mathrm{bq}^{0}=\mathrm{Z}^{0} \\
& \text { ou } \mathrm{v}_{y}=\mathrm{v}_{\mathrm{o}} \mathrm{y}-\mathrm{v}_{t} \mathrm{~s}
\end{aligned}
$$

ou seja, quando temos um produto agrícola, o valor verdadeiro de y é igual ao somatório dos valores ótimos dos seus elementos $\left(\mathrm{y}_{\mathrm{i}}\right)$ menos o valor-sombra das terras. Considerando apenas os valores-trabalho individuais ótimos das mercadorias (sem excluir o valor-sombra da terra), o somatório é, em geral, superior ao valortrabalho da mercadoria composta y. Ou seja, nesse caso teremos

$$
v_{y} \leq \sum v_{o i} y_{i}
$$

Este resultado é diferente do que Morishima \& Catephores (1980) apresentam $\left(v_{y}=\sum v_{o i} y_{t}\right)$, e é relevante, uma vez que indica que os valores ótimos, como os valores verdadeiros individuais, não são aditivos na presença de técnicas alternativas, como é o caso de um sistema econômico com um produto agrícola produzido em terras com diferentes qualidades. ${ }^{7}$

É interessante notar que $\mathrm{v}_{\mathrm{o}} \mathrm{y}$ representa o valor-trabalho de y se toda a produção ocorresse no pior tipo de terra [a terra marginal para o problema (7)-(9)].

\section{A mensuração da exploração ${ }^{8}$}

No capítulo X, volume I, de O Capital, Marx discutiu três maneiras de quantificar a exploração que pressupunha serem equivalentes.

A primeira é dada pela razão entre o trabalho excedente e o trabalho necessário. Suponhamos que um assalariado trabalhe $\mathrm{T}$ horas por período e receba uma cesta de bens, $\mathrm{d}$, para sua manutenção. Consideremos, ainda, que existam $\mathrm{N}$ tra-

\footnotetext{
${ }^{6}$ Sobre o teorema da dualidade, ver Ravindran et al. (1987).

${ }^{7}$ Ver Morishima \& Catephores (1980), p. 68, nota 29 (referida na p. 46).

${ }^{8}$ Esse item está baseado em Morishima \& Catephores (1980, pp. 46-53).
} 
balhadores homogêneos. Então, Nd é o vetor com os montantes de bens consumidos por todos os trabalhadores do sistema. Podemos obter o valor-trabalho desse vetor, $v_{N d}$ ou seja, o valor do trabalho necessário, substituindo y por $\mathrm{Nd}$ na equação (2) do problema (1) - (4). Com isso, obteremos a seguinte relação entre trabalho excedente e trabalho necessário:

$$
e_{1}=\frac{T N-v_{N d}}{v_{N d}}
$$

Uma segunda maneira de mensurar a exploração, segundo Marx, consiste em tomar por base apenas um trabalhador. Seja $v_{d}$ o valor-trabalho da cesta de consumo de um trabalhador, obtido substituindo-se y por d na equação (2) do problema (1)-(4). Então, a razão entre os trabalhos não-remunerado e remunerado por trabalhador é:

$$
e_{2}=\frac{T-v_{d}}{v_{d}}
$$

Por fim, segundo Morishima \& Catephores (1980), a terceira maneira de quantificar a exploração é a razão entre a mais-valia e o capital variável:

$$
e_{3}=\frac{v_{y}-v_{N d}}{v_{N d}}
$$

em que tanto $v_{Y} \operatorname{como} v_{N d}$ são obtidos resolvendo problemas como (1)-(4).

Como foi dito anteriormente, para Marx as três razões acima eram equivalentes, o que efetivamente ocorre na ausência de produção conjunta e técnicas alternativas. Para Morishima \& Catephores (1980), em geral tem-se $e_{1}=e_{2} \geq e_{3}$. Segundo eles, a desigualdade deve-se ao fato de que o vetor de produção, em uma economia capitalista maximizadora dos lucros, não corresponde, necessariamente, ao vetor que busca a 'utilização eficiente do trabalho', no problema de minimização acima. Nesse caso, podemos, então, ter $T N \geq v_{y}$.

No entanto, quando analisamos um sistema econômico com produção agrícola em terras com diferentes qualidades, teremos $e_{2} \geq e_{1} \geq e_{3}$, porque o produto necessário para uma única unidade de trabalho pode ser obtido na terra mais produtiva em termos da quantidade de trabalho por unidade de produto. Em outras palavras, o produto necessário, sendo menor, poderá ser produzido na terra mais produtiva, de maneira que

$$
\frac{v_{N d}}{N} \geq v_{d^{\prime}}
$$

Seguindo Morishima (1974), vamos utilizar e $e_{1}(=\mathrm{cr})$ como a medida do grau de exploração no sistema econômico ou como a taxa de mais-valia, uma vez que fazemos os cálculos da exploração sobre o tempo total de trabalho utilizado na economia. 


\section{OS PREÇOS DE PRODUÇÃO}

De início, vamos indicar por $\mathrm{t}$ um vetor-linha com $n_{z}$ elementos $\left(t_{m}\right.$, com $\mathrm{m}=$ $1, \ldots, n_{z}$, que são a renda da terra por unidade de área em cada tipo de terra. Os preços de produção constituem o vetor-linha $\mathrm{p} \operatorname{com} n_{a}+1$ elementos (os $n_{\mathrm{a}}$ preços das mercadorias industriais e o preço da mercadoria agrícola). Sendo $r$ a taxa de lucro e $w$ o salário, o modelo sraffiano-marxista em termos de preços de produção, com terras de diferentes qualidades, pode ser representado como segue:

$$
(\mathrm{pA}+\mathrm{b} w)(1+r)+[0 \mathrm{tH}]=\mathrm{pB}
$$

No modelo marxista, o salário real é previamente determinado por aspectos históricos, econômicos e institucionais, podendo ser representado por um vetor coluna de mercadorias d, com $n_{a}+1$ elementos, de maneira que $w=$ pd. Então, a equação (15) fica:

$$
(\mathrm{pA}+\mathrm{pdb})(1+r)+[0 \mathrm{tH}]=\mathrm{pB}
$$

Temos uma equação adicional associada ao fato de que na terra marginal não há renda:

$$
\prod_{m=1}^{n_{z}} t_{m}=0
$$

com $t_{m} \geq 0$. Vamos admitir que a terra marginal é a k-ésima, isto é, $t_{k}=0$, com $k \in\left(1, \ldots, n_{z}\right)$

O sistema acima possui $\mathrm{n}_{a}+n_{z}+2$ incógnitas (os $\mathrm{n}_{a}$, preços das mercadorias industriais, as $n_{z}$ rendas da terra, a taxa de lucro e o preço da mercadoria agrícola) e $n_{a}+n_{z}+1$ equações. Escolhendo uma mercadoria como numerário, obtém-se, em geral, urna solução única para o sistema.

A renda é uma decorrência da escassez. Nesse sistema supomos que a demanda por cereal só pode ser atendida cultivando-se todas as qualidades de terra. As diferentes qualidades de terra recebem renda porque são escassas, com exceção da terra de pior qualidade, que é abundante e, portanto, não recebe renda.

A determinação da margem extensiva ou da terra marginal pode ser analisada de maneira análoga à questão da escolha da técnica. A terra marginal é aquela que corresponde à técnica menos eficiente ou de máximo custo, ou, ainda, de mínima taxa de lucro, para um dado salário real (d).

Obtemos os preços de produção e a taxa de lucro, para um dado vetor d, através de um subsistema preços de produção-taxa de lucro. Esse subsistema coincide com um sistema de produção simples e possui solução economicamente significativa. Sendo formado por $n_{a}$ equações referentes ao sistema industrial mais uma equação referente à mercadoria agrícola, onde supomos que $t_{m}=0^{9}$. Admitindo que

\footnotetext{
${ }^{9}$ Sobre os modelos sraffianos com produção simples, ver Pasinetti (1977 e Quadrio-Curzio (1980).
} 
não há produção conjunta e que os coeficientes em $\mathrm{A}$ e b se referem à produção de uma unidade de cada mercadoria, temos:

$$
\left(\mathrm{p}_{\mathrm{m}} \mathrm{A}_{m}+\mathrm{p}_{m} \mathrm{db}_{m}\right)\left(1+\mathrm{r}_{m}\right)=\mathrm{p}_{m}
$$

onde $m=1, \ldots, n_{z}$. Neste caso, a matriz quadrada $\mathrm{A}_{m}$ tem dimensões $\left(n_{a}+1\right) \mathrm{x}\left(n_{a},+1\right)$ e os vetores $\mathrm{P}_{m}, \mathrm{~b}_{m}$ e d têm $n_{a}+1$ elementos.

Assim, considerando a mercadoria agrícola como básica, dado um vetor d, a solução para o sistema muda para cada $t_{\mathrm{m}}$ igual a zero. Em geral, haverá $n_{z}$ soluções. A terra $k$ menos eficiente, denominada terra marginal, será aquela que, para o mesmo vetor $\mathbf{d}$, gerar a menor taxa de lucro. Então,

$$
r_{k} \leq r_{m}
$$

com $k \in\left(1, \ldots, n_{z}\right)$ Retirando a terra marginal $(\mathrm{k})$, as $n_{z}-1$ equações restantes do setor agrícola formam um outro subsistema, denominado subsistema renda. Cada equação desse subsistema possui apenas uma incógnita: a renda. Obtidos $r_{k}$ e $\mathrm{p}_{k}$, cujo último elemento é o preço do cereal $\left(\mathrm{P}_{\mathrm{z}}\right)$, a renda por unidade de área da terra tipo $m$ é:

$$
t_{m}=\frac{p_{z}-\left(p_{k} a_{m}+\mathrm{p}_{k} \mathrm{~d} b_{j}\right)\left(1+r_{k}\right)}{h_{m}}
$$

onde $\mathbf{a}_{m}$ é a coluna de $\mathbf{A}$ e $b_{i}$ é o elemento de $\mathbf{b}$ correspondente à produção na terra tipo $m$.

\section{UM EXEMPLO NUMÉRICO, ${ }^{10}$ VALORES-TRABALHO E TAXA DE EXPLORAÇÃO}

Nesta seção, será apresentado um sistema que supõe uma economia com duas mercadorias: uma industrial e uma agrícola. Vamos considerar dois tipos de terra, indicados por $\alpha$ e $\beta$, cada um com sua técnica própria de cultivo. Admite-se que a utilização de apenas um dos dois tipos de terra é insuficiente para atender toda a demanda da economia por cereal, mas não há necessidade de usar toda a área disponível das duas qualidades de terra. Vamos considerar, ainda, que a mercadoria agrícola é básica.

Sejam $\mathrm{Q}_{i j}$ e $L_{j}$ respectivamente, a quantidade da mercadoria $i$ e a quantidade de trabalho utilizadas na atividade $j$, e $H_{m}$, a área de terra do tipo $m$ que é cultivada, com $m=\alpha$ ou $\beta$. Então, os processos de produção para as duas mercadorias são completamente descritos pelas seguintes expressões:

\footnotetext{
${ }^{10} \mathrm{O}$ exemplo numérico utilizado nesta seção foi desenvolvido por Montani (1975) e analisado por Hoffmann \& Venter (1990). Salienta-se que ele foi aplicado apenas ao modelo sraffiano e não ao sraffianomarxista, aqui desenvolvido.
} 
$\mathrm{Q}_{11}$ merc.1 $\oplus \mathrm{Q}_{21}$ merc.2 $\oplus L_{1}$ trabalho $\rightarrow \mathrm{Q}_{1}$ merc. 1

$\mathrm{Q}_{12}$ merc.1 $\oplus \mathrm{Q}_{22}$ merc.2 $\oplus L_{2}$ trabalho $\oplus H_{1}$ terra $\alpha \rightarrow \mathrm{Q} 2$ merc.2

$\mathrm{Q}_{13}$ merc.1 $\oplus \mathrm{Q}_{23}$ merc.2 $\oplus L_{3}$ trabalho $\oplus H_{2}$ terra $\beta \rightarrow \mathrm{Q} 3$ merc.2

onde “ $\oplus$ ” significa “combinado com" e " $\rightarrow$ " significa "para produzir".

Considerando $a_{i j}=\frac{Q_{l j}}{Q_{j}}, b_{l}=\frac{L_{l}}{Q_{l}} e h_{l}=\frac{H_{l}}{Q_{l}}$, temos:

$$
\begin{aligned}
\mathrm{A} & =\left[\begin{array}{lll}
a_{11} & a_{12} & a_{13} \\
a_{21} & a_{22} & a_{23}
\end{array}\right] \\
\mathrm{b} & =\left[\begin{array}{lll}
b_{1} & b_{2} & b_{3}
\end{array}\right] \mathrm{e} \\
\mathrm{H} & =\left[\begin{array}{ll}
h_{a} & 0 \\
0 & h_{\beta}
\end{array}\right]
\end{aligned}
$$

Sejam, ainda, o vetor $\mathbf{d}$ e a matriz $\mathbf{B}$ dados como se segue:

$$
\begin{aligned}
& \mathrm{d}=\left[\begin{array}{l}
d_{1} \\
d_{2}
\end{array}\right] \\
& \mathrm{B}=\left[\begin{array}{lll}
1 & 0 & 0 \\
0 & 1 & 1
\end{array}\right]
\end{aligned}
$$

Vamos analisar o seguinte exemplo numérico:

4 merc. $1 \oplus 1$ merc. $2 \oplus 0,5$ trabalho $\rightarrow 10$ merc. 1

0,5 merc. $1 \oplus 2$ merc. $2 \oplus 0,15$ trabalho $\oplus 1$ terra $\alpha \rightarrow 4,2$ merc. 2

2,5 merc. $1 \oplus 0,4$ merc. $2 \oplus 0,4$ trabalho $\oplus 1$ terra $\beta \rightarrow 5,4$ merc. 2

Podemos ler a equação (30), por exemplo, como: 0,5 unidade da mercadoria industrial combinadas com 2 unidades da mercadoria agrícola, 0,15 unidade de trabalho e 1 unidade de área da terra de qualidade a produzem 4,2 unidades do produto agrícola.

Verifica-se que o produto líquido do sistema é:

$$
y=\left[\begin{array}{l}
3,0 \\
6,2
\end{array}\right]
$$

Vamos admitir que o consumo dos trabalhadores por unidade de trabalho seja:

$$
d=\left[\begin{array}{l}
2,2 \\
5,5
\end{array}\right]
$$


Vamos examinar, inicialmente, o cálculo dos valores verdadeiros. Admitimos que haja disponibilidade de apenas uma unidade de cada tipo de terra, ou seja,

$$
s=\left[\begin{array}{l}
s_{a} \\
s_{\beta}
\end{array}\right]=\left[\begin{array}{l}
1 \\
1
\end{array}\right]
$$

Nesse caso, o problema de programação linear descrito nas equações (1)-(4) fica:

Minimizar $\left[\begin{array}{lll}b_{1} & b_{2} & b_{3}\end{array}\right]\left[\begin{array}{l}q_{1} \\ q_{2} \\ q_{3}\end{array}\right]$

sujeito a

$$
\begin{array}{r}
{\left[\begin{array}{ccc}
1-a_{11} & -a_{12} & -a_{13} \\
-a_{21} & 1-a_{22} & 1-a_{23}
\end{array}\right]\left[\begin{array}{l}
q_{1} \\
q_{2} \\
q_{3}
\end{array}\right] \geq\left[\begin{array}{l}
y_{1} \\
y_{2}
\end{array}\right]} \\
{\left[\begin{array}{ll}
h_{\alpha} & 0 \\
0 & h_{\beta}
\end{array}\right]\left[\begin{array}{l}
q_{2} \\
q_{3}
\end{array}\right] \leq\left[\begin{array}{l}
s_{\alpha} \\
s_{\beta}
\end{array}\right]}
\end{array}
$$

com $q_{i} \geq 0$, para $i=1,2$ e 3 .

Para o exemplo numérico, o valor-trabalho verdadeiro do produto líquido y dado na equação (32) é 1,05 , que é minimizado para

$$
\mathrm{q}^{0}=\left[\begin{array}{l}
10 \\
4,2 \\
5,4
\end{array}\right]
$$

Nota-se que, no caso dessa definição, o valor da mercadoria composta y é, por construção, igual ao total de trabalho empregado (bq), de acordo com a equação ( 1 ). Pode-se verificar que o valor individual calculado pelo primeiro método sugerido por Morishima \& Catephores (1980), considerando um produto líquido constituído por apenas uma unidade da mercadoria industrial, é de 0,09842, e por apenas uma unidade da mercadoria agrícola, 0,09055. Utilizando esses valores podemos verificar a validade da equação (6), ou seja,

$$
v_{y}=1,05>\sum v_{y l} y_{l}=0,85669
$$

O total obtido utilizando os valores verdadeiros individuais é menor porque esses são determinados considerando a produção de cereal na terra mais produtiva, ao passo que a obtenção de todo o produto líquido exige que parte do cereal seja produzida na terra menos produtiva.

Os valores-trabalho ótimos são obtidos pelo seguinte problema de programação linear [ver equações (7)-(9)]: 


$$
\begin{aligned}
& \text { Maximizar } Z=\left[\begin{array}{lll}
v_{o a} & v_{o z}-v_{t a}-v_{t \beta}
\end{array}\right]\left[\begin{array}{c}
y_{a} \\
y_{z} \\
s_{a} \\
s_{\beta}
\end{array}\right] \\
& {\left[\begin{array}{ll}
v_{o a} & v_{o z}
\end{array}\right]\left[\begin{array}{lll}
1-a_{11} & -a_{12} & -a_{13} \\
-a_{21} & 1-a_{22} & 1-a_{23}
\end{array}\right]-\left[0\left[\begin{array}{ll}
-v_{t a} & -v_{t \beta}
\end{array}\right]\left[\begin{array}{ll}
h_{a} & 0 \\
0 & h_{\beta}
\end{array}\right]\right] \leq\left[\begin{array}{lll}
b_{1} & b_{2} & b_{3}
\end{array}\right]} \\
& \mathrm{v}_{0} \geq \mathrm{e} 0 \text { e } \mathrm{v}_{t} \geq 0
\end{aligned}
$$

Pode-se verificar que os valores-trabalho ótimos para as mercadorias industrial e agrícola são, respectivamente, 0,10545 e 0,13273. Além disso, o valor-sombra da terra a é igual a 0,08927 e o da terra 'igual a zero. De acordo com o teorema da dualidade, obtemos:

$$
\mathrm{bq}^{\mathrm{o}}=1,05=\mathrm{Z}^{\mathrm{o}}
$$

Além disso, podemos observar que ocorre a desigualdade prevista na equação (11):

$$
v_{y}=1,05<\sum v_{o i} y,=1,13927
$$

Os valores individuais ótimos correspondem ao tempo de trabalho necessário para produzir uma unidade adicional de cada mercadoria. Como a terra $\alpha$, mais produtiva, já está totalmente utilizada, esses valores são condicionados pela produtividade menor obtida na terra $\beta$ Por isso, o valor total do produto líquido calculado com base nos valores individuais ótimos é maior do que o valor verdadeiro do produto líquido, em cujo cálculo se leva em consideração que parte do cereal pode ser produzido na terra mais produtiva.

Tendo em vista calcular as taxas de exploração dadas pelas equações (12)-(14), vamos determinar o trabalho necessário para produzir o consumo dos trabalhadores. Com $N=1,05$ e d dado na equação (33), o consumo total dos trabalhadores é de:

$$
N d=\left[\begin{array}{l}
2,31 \\
5,775
\end{array}\right]
$$

Resolvendo o problema de programação linear (35)-(37) para um produto líquido igual a $\mathrm{Nd}$, obtemos:

$$
v_{\mathrm{Nd}}=0,92083
$$

Segue-se que:

$$
e_{1}=\frac{T N-v_{N d}}{v_{N d}}=\frac{1,05-0,92083}{0,92083}=0,1403
$$

Uma vez que nesse caso o trabalho empregado (TN) é igual ao valor verdadeiro do produto líquido da economia $\left(v_{\mathrm{y}}\right)$, temos $e_{3}=e_{1}$. 
Resolvendo o problema de programação linear (35)-(37) e considerando a obtenção de um produto líquido igual ao consumo de uma unidade de trabalho (d), obtemos:

$$
\begin{aligned}
& v d=0,87273 \\
& \mathrm{e}, \text { consequentemente, } \\
& e_{2}=\frac{T-v_{d}}{v_{d}}=\frac{1-0,87273}{0,87273}=0,1458
\end{aligned}
$$

Esse resultado mostra a possibilidade de termos $e_{2}>e_{1}$, como já foi assinalado na seção 2.2. A medida mais adequada do grau de exploração é, certamente, o valor de $e_{1}$ onde se considera como mais-valia a diferença entre o tempo de trabalho efetivamente empregado e o tempo de trabalho (mínimo ) necessário para produzir o consumo de todos os trabalhadores.

No cálculo de $v_{\mathrm{Nd}}$ admite-se que a economia seja reestruturada para obter, como produto líquido, apenas o consumo dos trabalhadores, o que fará com que maior proporção do cereal possa ser produzido na terra mais produtiva, reduzindo o tempo médio de trabalho por unidade produzida.

Os resultados obtidos mostram que para uma economia com setor agrícola em que são cultivados vários tipos de terra os valores-trabalho devem ser calculados como os valores verdadeiros de Morishima, apesar de surgirem desigualdades não previstas por Morishima \& Catephores.

É interessante notar que o valor-sombra da terra $\alpha\left[\mathrm{v}_{t \alpha}=0,08927\right.$ na solução do problema (40)-(42)], que pode ser associado à renda da terra, não aparece como parcela da mais-valia [o numerador da equação (47) é igual a 0,12917], mas como um valor-trabalho hipotético acima do valor-trabalho do produto líquido $(1,05)$, que só existiria, efetivamente, se não houvesse a terra de tipo a e todo o cereal tivesse de ser produzido em terras do tipo $\beta$. Isso é perfeitamente coerente com a noção marxista de que a terra contribui para produzir os valores de uso, mas não gera valortrabalho. A renda da terra, como uma parcela do valor monetário do excedente, só aparece no domínio dos preços de produção.

\section{PREÇOS DE PRODUÇÃO E RENDA DA TERRA NO EXEMPLO NUMÉRICO}

Vamos considerar, agora, a determinação da terra marginal e da renda da terra no sistema sraffiano-marxista em termos dos preços de produção, de acordo com a equação (15), ou seja,

$$
(\mathrm{pA}+b w)(1+r)+[\mathrm{O} \mathrm{tH}]=\mathrm{pB}
$$

Os preços de produção estão representados por

$$
\mathrm{P}=\left[P_{a} P_{z}\right]
$$


e as rendas da terra por

$\mathbf{t}=\left[t_{\alpha} t_{\beta}\right]$

As matrizes A, b, H, d e B foram descritas nas equações (24) a (28).

As incógnitas desse sistema são a taxa de lucro $(\mathrm{r})$, os preços de produção $\left(p_{a}\right.$ e $\left.P_{z}\right)$ e as rendas da terra $\left(t_{a}\right.$ e $\left.t_{\beta}\right)$.

Admitindo que a terra $\alpha$ seja a marginal, temos $t_{\alpha}=\mathrm{O}$. Nesse caso, o subsistema preços de produção-taxa de lucro pode ser representado por

$$
\begin{aligned}
& \left(\mathbf{p}_{\alpha} \mathrm{A}_{\alpha}+\mathbf{p}_{\alpha} \mathrm{db}_{\alpha}\right)\left(1+r_{\alpha}\right)=\mathrm{P}_{\alpha} \\
& \text { onde } \mathrm{A}_{\alpha}=\mathrm{A}_{\alpha}=\left[\begin{array}{ll}
a_{11} & a_{12} \\
a_{21} & a_{22}
\end{array}\right] \\
& \mathrm{eb}_{\alpha}=\left[b_{1} b_{2}\right]
\end{aligned}
$$

Este sistema possui como incógnitas $p_{\alpha}, p_{z}$ e $r_{\alpha}$ e apenas duas equações. Tomando a mercadoria agrícola como numerário, temos $p_{z}=1 \mathrm{e}$

$$
\mathrm{p}=\left[\begin{array}{ll}
p_{a} & 1
\end{array}\right]
$$

Vamos considerar a matriz de coeficientes técnicos aumentada:

$$
\mathrm{A}_{\alpha}^{+}=\mathrm{A}_{\alpha}+\mathrm{db}_{\alpha}
$$

A taxa de lucro desse sistema pode ser obtida a partir da raiz característica máxima $\left(\lambda_{\alpha}\right)$ da seguinte equação característica:

$$
\left|\mathrm{A}_{\alpha}^{+}-\lambda_{\alpha} 1\right|=0
$$

Temos que

$$
\lambda_{\alpha}=\frac{1}{1+r_{\alpha}}
$$

e, com isso, obtemos a taxa de lucro.

Considerando o exemplo numérico descrito anteriormente, a partir da equação (58) obtemos as raízes 0,87542 e 0,30720. Então, a raiz característica máxima é $\lambda_{\alpha}=0,87542$ e, consequentemente, $r_{\alpha}=0,14231$.

Se a terra $\beta$ for a menos eficiente, temos o seguinte subsistema preços de produção-taxa de lucro:

$$
\begin{aligned}
& \left(\mathrm{p}_{\beta} \mathrm{A}_{\beta}+\mathrm{p}_{\beta} \mathrm{db}_{\beta}\right)\left(1+\mathrm{r}_{\beta}\right)=\mathrm{p}_{\beta} \\
& \text { onde } \mathrm{A}_{\beta}=\left[\begin{array}{ll}
a_{11} & a_{13} \\
a_{21} & a_{23}
\end{array}\right]
\end{aligned}
$$




$$
\text { e } \quad \mathrm{b}_{\beta}=\left[\begin{array}{ll}
b_{1} & b_{3}
\end{array}\right]
$$

Para o mesmo exemplo numérico, obtemos, similarmente, $\lambda_{\beta}=0,98043$ e $r_{\beta}=$ 0,01996. Com isso, verifica-se que

$$
r_{\alpha}>r_{\beta}
$$

e, portanto, a terra de qualidade $\beta$ é a marginal.

O vetor-linha com os preços de produção é um vetor característico à esquerda de $\mathrm{A} / \mathrm{J}$, correspondente à raiz característica máxima. Então,

$$
\begin{aligned}
& \mathrm{p}_{\beta} \mathrm{A}_{\beta}^{+}=\lambda_{\beta} \mathrm{p}_{\beta} \\
& \text { ou } \mathrm{p}_{\beta}\left(\mathrm{A}_{\beta}^{+}-\lambda_{\beta} 1\right)=0
\end{aligned}
$$

Para o exemplo numérico, $\operatorname{com} p_{z}=1$, obtemos:

$$
\mathrm{p}_{\beta}=[0,797141]
$$

Determinamos o salário em unidades monetárias em termos da mercadoria agrícola, como segue:

$$
w_{\beta}=\mathrm{p}_{\beta} \mathrm{d}=7,2537
$$

Podemos, ainda, obter a renda na terra $a$, de acordo com a equação (20):

$$
t_{\alpha}=\frac{1-\left(p_{\beta} \mathrm{a}_{\alpha}+b_{2} w_{\beta}\right)\left(1+r_{\beta}\right)}{h_{\alpha}}=0,6438
$$

Vamos, agora, estudar os efeitos de mudanças no vetor d. Para cada vetor d fixado exogenamente, obtemos uma nova solução para o sistema acima. Podemos modificar o salário real multiplicando o vetor d por uma constante 0 . Lembrando a equação (33), temos:

$$
\mathrm{d}^{*}=\theta \mathrm{d}=\theta \begin{aligned}
& 2,2 \\
& 5,5
\end{aligned}
$$

Dessa maneira, mantemos a proporcionalidade na cesta de mercadorias consumida por um trabalhador no sistema econômico. Quando $\theta=1$, temos o sistema original. A cesta de mercadorias, em termos físicos, que compõe o salário dos trabalhadores pode ser modificada, hipoteticamente, de um extremo em que é nula, quando a taxa de lucro alcança seu valor máximo, a outro extremo, em que absorve todo o produto líquido de pelo menos uma mercadoria do sistema.

Com isso, obtemos duas relações entre $\theta$ e a taxa de lucro: uma para o caso em que consideramos a terra ex como marginal e a outra para o caso em que fazemos a mesma suposição para a terra $\beta$, como mostra a Figura 1a. Essas relações são decrescentes, pois um aumento do salário real representa uma redução na taxa de lucro. A taxa de lucro máxima é obtida fazendo $\mathrm{d}^{*}=0$, o que implica $w=0$, e 
é igual a 0,81 na terra $\alpha$ e 0,97 na terra $\beta$. O valor mínimo para a taxa de lucro $\left(r_{a}\right.$ ou $r_{\beta}$ igual a 0$)$ é alcançado quando a raiz característica máxima da matriz de coeficientes técnicos aumentada $\left(\mathbf{A}_{\alpha}^{+}\right.$ou $\left.\mathbf{A}_{\beta}^{+}\right)$assume o valor 1.

As duas relações se interceptam quando $r^{a}=r^{\beta}=0,476$ e $\theta=0,376$. Quando $r$ está entre 0 e 0,476 , a terra $\alpha$ é a mais eficiente e, quando temos $0,476<r<0,81$, a terra $\beta$ passa a ser a mais eficiente. Isso ocorre porque na terra $\beta$ utiliza-se uma técnica que é mais intensiva em trabalho que a técnica empregada na terra $\alpha$. Assim, enquanto existe uma participação pequena do salário real no excedente, a técnica $\beta$ é mais "barata", mas quando essa participação vai aumentando (valores crescentes de $\theta$ ) essa técnica vai se tornando relativamente mais "cara". O contrário ocorre na terra $a$, com uma técnica menos intensiva em trabalho. Desse modo, para uma baixa participação do salário real no excedente, esta técnica é menos eficiente. Quando essa participação aumenta, a técnica $\alpha$ torna-se, paulatinamente, mais eficiente porque o trabalho ficou mais "caro", e ela utiliza menos desse insumo do que a técnica $\beta$.

A relação entre a taxa de lucro e o salário está apresentada na Figura 1 b. Como estamos assumindo que a demanda por cereal não pode ser atendida com o cultivo de uma única qualidade de terra, então a máxima taxa de lucro dessa economia será igual a 0,81 (ponto $\mathrm{C}$ ), determinada na terra $\alpha$. Isso ocorre porque maior taxa de lucro inviabilizará a produção nessa terra. O ponto de mudança de eficiência das técnicas nesse sistema ocorre quando $r=0,476$ (ponto $B$ ). Nos pontos $A, B$ e $C$ o salário assume, respectivamente, os valores 7,53, 2,77 e zero. Com isso, a fronteira tecnológica para este sistema é representada pela curva $A B C$, como mostra a Figura $1 \mathrm{~b}$.

$\mathrm{Na}$ Figura 1a observa-se que o sistema econômico se torna inviável para ft 1,04 , uma vez que não é possível cultivar as duas qualidades de terra. Para $\theta \geq 1,04$ o cultivo da terra $\beta$ deixa de ser lucrativo e, portanto, não é possível atender toda a demanda.

A Figura 1c mostra que, para valores de $r$ entre 0 e 0,476 , a terra $a$, mais eficiente, tem uma renda positiva e, para valores de $r$ entre 0,476 e 0,81 , a terra $\beta$, que passa a ser a mais eficiente, é a que tem renda positiva. Quando $r=0,476$ não existe renda da terra, pois os dois tipos de terra são igualmente eficientes.

$\mathrm{Se}$, ao contrário, admitirmos que a quantidade de cereal que deve ser produzida seja pequena, de maneira que cultivando apenas parte de uma das qualidades de terra possamos satisfazer as necessidades da sociedade, então será cultivada apenas a terra com a técnica mais eficiente. Então, para $0<r<0,476$ será cultivada apenas a terra $a$ e quando $0,476<r<0,81$, apenas a terra $\beta$. Assim, a fronteira tecnológica seria dada pela parte externa da Figura $1 b$, ao contrário do que foi visto acima, em que a fronteira é composta pelas partes internas dessa figura. Uma consequência dessa suposição sobre a demanda é que como nenhuma terra é escassa não existe renda da terra em nenhum ponto da relação $w$-r.

Podemos concluir que a ordem de eficiência das terras não é naturalmente estabelecida, mas depende da distribuição da renda entre salários e taxa de lucro, além da demanda total por cereal. Até mesmo o fato de determinado tipo de terra 
Figura 1: Relações $\theta-r, w-r$ e $t-r$ para um modelo sraffiano-marxista com terras de diferentes qualidades

a)

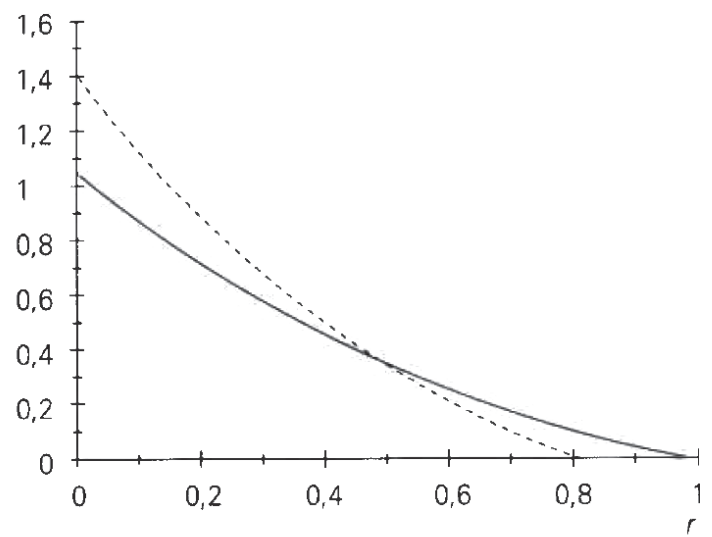

b)

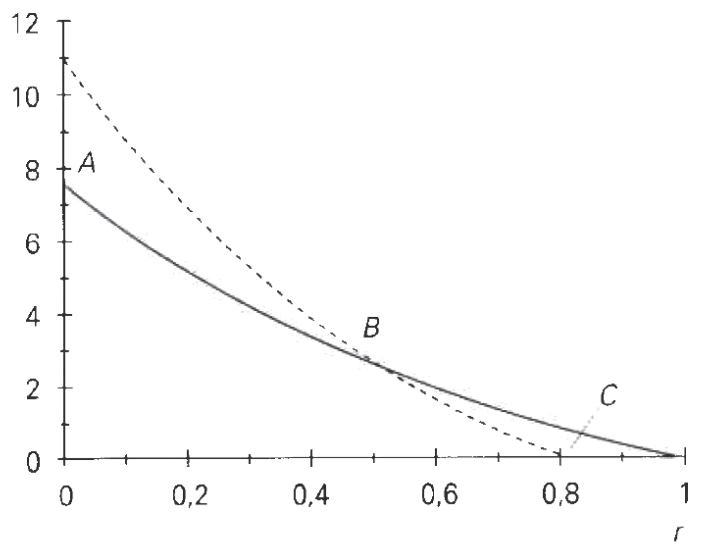

c) $t 3$

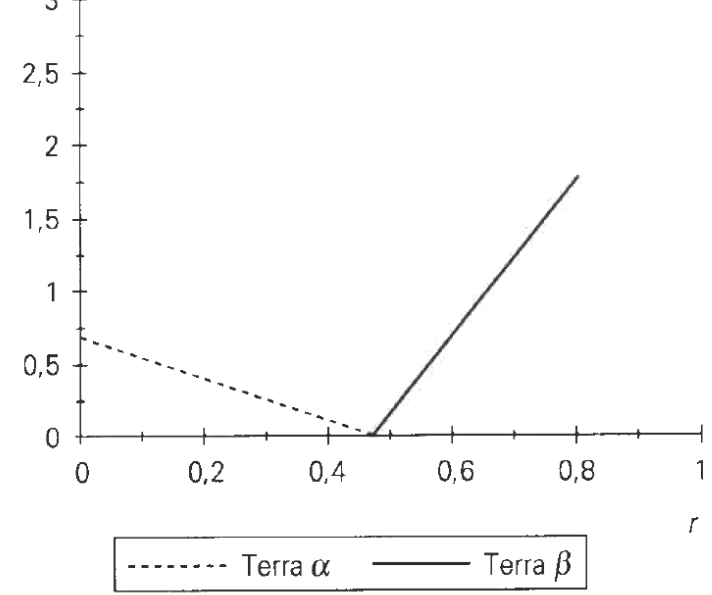


ser escasso ou não depende da distribuição da renda entre salários e lucros. Dadas as quantidades dos bens que devem ser produzidas, os diversos valores do salário e da taxa de lucro é que vão determinar se uma qualidade de terra específica é escassa ou abundante.

Este fenômeno, discutido por Sraffa, não foi analisado por Ricardo ou Marx. Ricardo acreditava que existia uma hierarquia das terras de acordo com a fertilidade naturalmente estabelecida e inalterada, dadas as quantidades de capital. Marx compartilhava da mesma opinião sobre a ordem de fertilidade das terras, para iguais aplicações de capital nas diferentes qualidades de terra, embora tenha ressaltado que essa ordem era alterada pela evolução da tecnologia agrícola.

Caso se considere que nas diferentes qualidades de terra se utilize a mesma quantidade dos mesmos meios de produção e de trabalho, por unidade de área, as relações $w-r$ passam a ser linhas paralelas. Portanto, não existiria mudança na ordem de eficiência das terras em decorrência de alterações na distribuição de renda, dada uma demanda por cereal.

Abandonando essas restrições, ou seja, considerando que terras diferentes são exploradas utilizando diferentes quantidades dos meios de produção e de trabalho, como faz Sraffa, essas conclusões de Ricardo e Marx não se aplicam.

\section{VARIAÇÃO DO CAPITAL VARIÁVEL E DA MAIS-VALIA}

Vamos continuar analisando o mesmo exemplo numérico, verificando como alterações no vetor $\mathrm{d}$ afetam o capital variável, a mais-valia e a taxa de mais-valia. Os valores-trabalho são calculados de acordo com o método de Morishima e as alterações no consumo dos trabalhadores são feitas conforme a equação (69), multiplicando-se o vetor d original por um escalar $\theta$.

A Figura 2a mostra como o capital variável $(V)$ cresce $\operatorname{com} \theta$. Nota-se que há uma mudança na inclinação dessa relação quando $\theta=0,34$. Isso ocorre porque para valores baixos de $\theta$ todo o cereal necessário pode ser produzido na terra $\alpha$, que é mais produtiva. A partir do ponto em que a quantidade necessária de cereal exige o uso da terra $\beta$ (passando a ser atuante, na resolução do problema de programação linear, a restrição relativa à disponibilidade da terra $\alpha$ ), a inclinação da relação $V-\theta$ aumenta.

O valor mínimo de $V$ ocorre quando $\theta$ é igual a zero e o valor máximo, quando todo o trabalho disponível é empregado na produção da cesta de consumo dos trabalhadores, nesse caso para $\theta=1,087$ e $V=1,009$.

A Figura $2 \mathrm{~b}$ apresenta a relação entre a taxa de mais-valia e o coeficiente $\theta$. A curva se aproxima do eixo das ordenadas como uma hipérbole retangular, pois se o vetor $\theta N d$ assumisse seu valor mínimo (nulo) o capital variável seria, também, igual a zero e a taxa de mais-valia tenderia para infinito. O valor mínimo da taxa de maisvalia ocorre quando $V$ atinge seu valor máximo. Esse valor mínimo pode ser ou não igual a zero, dependendo da composição do vetor d. Neste exemplo, para $\theta=1,087$ a taxa de mais-valia é igual a $4 \%(\sigma=0,04)$, mas para $\theta>1,087$ 
Figura 2: Relações $V-\theta \sigma-\theta$ e $\sigma-V$ para um modelo marxista com terras de diferentes qualidades

a) $\quad$

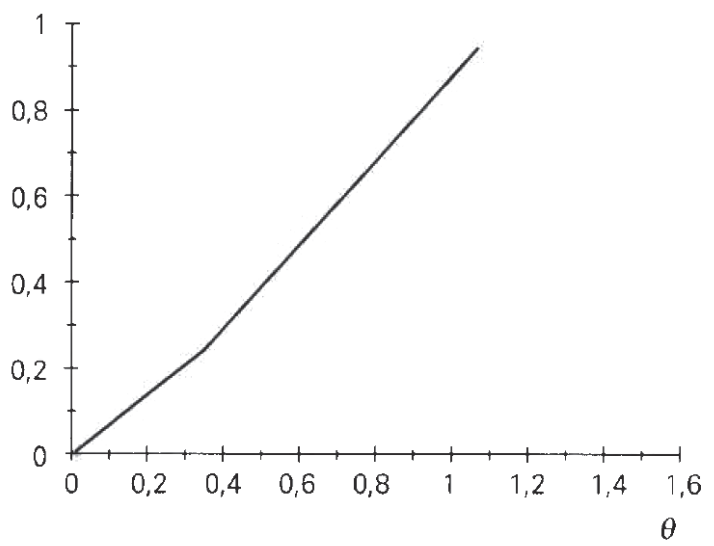

b) $\sigma \quad 60$

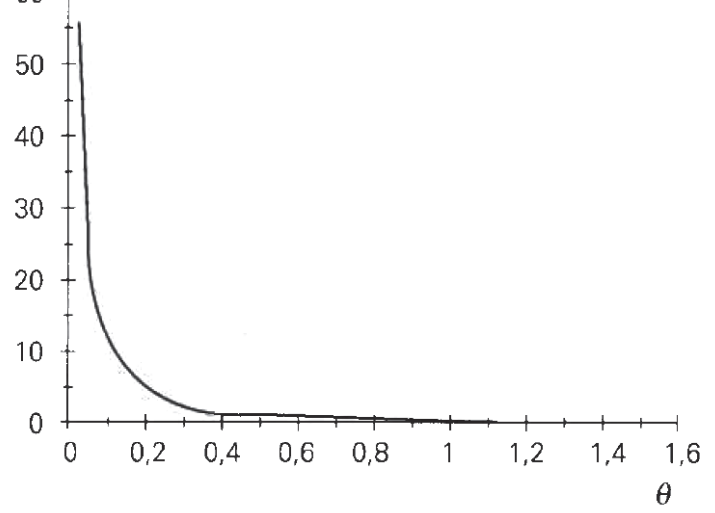

c) $\sigma$

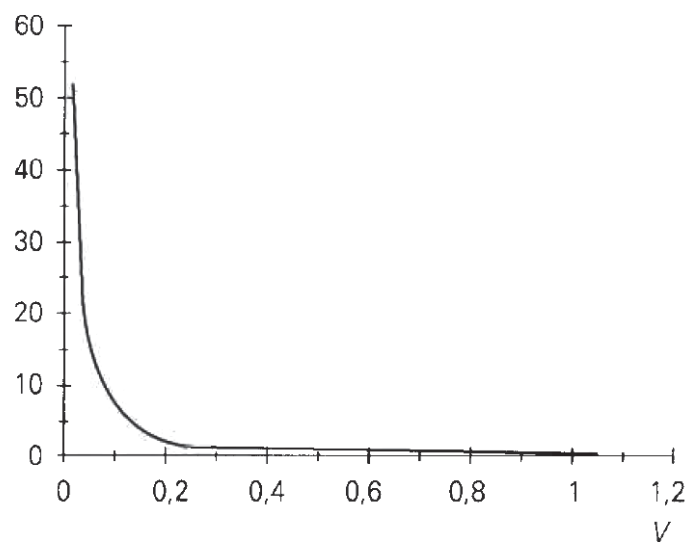


é impossível obter um produto líquido que seja suficiente para atender o consumo dos trabalhadores.

A Figura $2 \mathrm{c}$ mostra a relação entre a taxa de mais-valia e o capital variável. Essa relação é similar àquela existente na Figura 26, uma vez que substituímos $\theta$ pelo valor da cesta de mercadorias consumida por todos os trabalhadores do sistema, que possuem uma relação crescente entre si, como vimos na Figura 2a.

Para cada vetor $\mathrm{d}$, fixado exogenamente, podemos determinar tanto o sistema em termos de preços de produção quanto o sistema em termos de valores-trabalho e, além disso, estabelecer relações entre eles. Em outras palavras, partindo de um sistema em unidades físicas podemos ir dos preços de produção aos valores-trabalho ou, o contrário, dos valores-trabalho aos preços de produção.

A Figura 3 apresenta a relação entre a taxa de lucro vigente neste exemplo, ou seja, aquela referente à curva interna da Figura $1 \mathrm{a}$, e a taxa de mais-valia. Nota-se que essa relação é crescente e temos $\sigma>r$.

Ao examinar a Figura 1 vimos que o cultivo da terra $\beta$ deixa de ser lucrativo para $\theta 1,04$. Entretanto, a mais-valia é positiva para $\theta \leq 1,087$. Há, então, uma faixa de valores de $\theta$ para a qual a mais-valia é positiva, mas já não é possível a produção lucrativa. Isso corresponde, na Figura 3, ao fato de $r$ só ser positivo para $\sigma$ maior do que 0,09 . Conclui-se, portanto, que em um sistema econômico em que há escassez de algum tipo de terra a mais-valia positiva não é condição suficiente para que haja taxa de lucro positiva.

Figura 3: Relações $\sigma-r$ para um sistema sraffiano-marxista com terras de diferentes qualidades

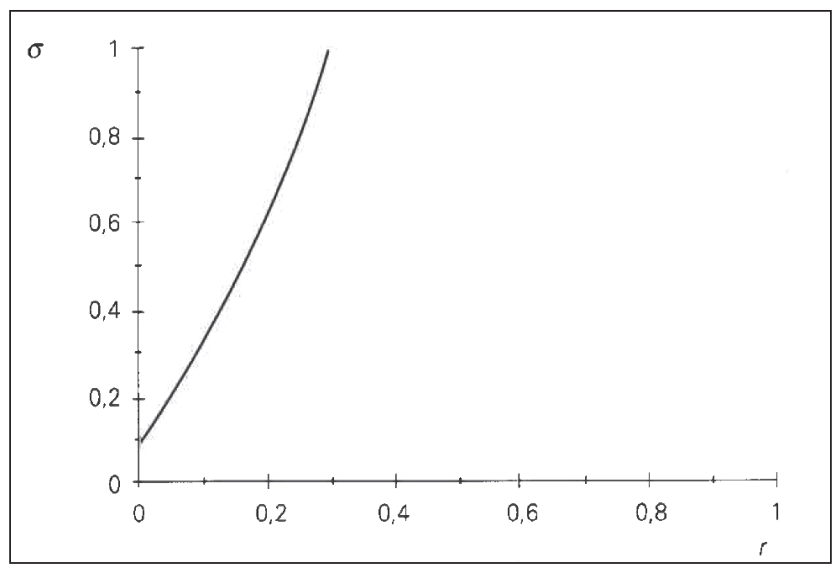

\section{CONSIDERAÇÕES FINAIS}

Com base na abordagem sraffiana e na contribuição de Morishima para a análise dos valores-trabalho, podemos observar que, havendo um setor agrícola com escassez de algum tipo de terra, a existência de uma taxa de mais-valia positiva é condição necessária, mas não suficiente para a existência de lucros positivos. Além disso, os valores verdadeiros são únicos e positivos. 
Verificamos, também, que três afirmações presentes no trabalho de 1980 de Morishima \& Catephores deixam de ser válidas em um sistema econômico com uso de terras escassas. Primeiro, a taxa de mais-valia calculada para um trabalhador individual pode ser maior do que aquela calculada para todos os trabalhadores do sistema, ou seja, $e_{2}>e_{1}$, diferentemente do que pensavam Morishima \& Catephores $\left(e_{2}=e_{1}\right)$ Segundo, nesse sistema econômico os valores ótimos, da mesma maneira que os valores verdadeiros individuais, não são aditivos. E, por fim, a soma dos valores individuais verdadeiros de cada mercadoria multiplicados pelas respectivas quantidades do seu produto líquido no sistema pode ser menor que o valor verdadeiro do produto líquido do sistema.

Os sistemas econômicos com o setor agrícola desenvolvidos neste trabalho representam uma extensão da abordagem sraffiana. Partindo de um sistema em unidades físicas, analisamos tanto sistemas em termos dos preços de produção quanto em termos dos valores- trabalho e, além disso, estabelecemos relações entre eles.

Por se tratar de uma abordagem formal, o modelo sraffiano-marxista em termos de preços de produção possui alguns aspectos não analisados por Marx. Diferentemente do que ocorre nas análises de Marx, nesses modelos doses desiguais de capital e trabalho podem ser aplicadas em terras com diferentes qualidades ou de mesma qualidade com diferentes técnicas de produção. No sistema de Sraffa, os preços relativos são determinados simultaneamente aos parâmetros distributivos (salário, taxa de lucro e renda da terra).

Uma conclusão obtida nesses modelos é que a ordem de eficiência das terras não representa uma característica naturalmente dada, mas depende da distribuição de renda e da demanda por cereal na economia. Ou seja, dada a demanda por cereal, a ordem de eficiência das terras pode mudar como consequência de uma mudança no salário real.

Cabe salientar que, contrastando com os pressupostos adotados pelos autores clássicos e por Marx, no enfoque sraffiano o salário não é mantido no nível de subsistência, permitindo uma análise mais realista do conflito distributivo em uma economia capitalista.

Uma contribuição de Marx sobre as condições necessárias para o aparecimento de renda de terra foi sua ênfase no fato de que a renda da terra só é paga para alguém que é o proprietário da terra. A terra constitui-se em um meio de produção não reproduzido, escasso e que pode ser apropriado.

A renda da terra é uma consequência da escassez da terra. Quando temos diferentes qualidades de terra, só recebem renda aquelas qualidades de terra totalmente cultivadas e, portanto, escassas, e quando temos uma terra de mesma qualidade na qual diferentes técnicas de cultivo podem ser empregadas, a renda aparece apenas quando duas técnicas estão sendo utilizadas lado a lado e essa terra homogênea é escassa.

Ressaltamos que a análise desenvolvida neste trabalho não tem sua aplicação limitada ao estudo de modelos que incluam o insumo terra, mas pode ser utilizada também para analisar outros meios de produção não produzidos, tais como os depósitos minerais. 
Quando consideramos sistemas com mais de duas qualidades de terra empregadas no cultivo do cereal, podemos verificar uma outra característica: a ordem de eficiência não corresponde, em geral, à ordem de rentabilidade das terras. Além disso, nesses modelos pode ocorrer o fenômeno do retorno de técnicas em sistemas com o setor agrícola. O retorno ou reeleição de uma técnica consiste no fato de uma técnica que já foi substituída por outra mais eficiente em função do aumento da taxa de lucro voltar a ser utilizada para valores ainda mais altos da taxa de lucro.

\section{REFERÊNCIAS}

FINE, B. (1979) “On Marx 's Theory of Agricultural Rent”. Economy and Society, v.8, n.3., August, p.241-278.

GAREGNANI, P. (1977) "Sobre a Teoria da Distribuição e do Valor em Marx e nos Economistas Clássicos”. In: GAREGNANI, P., STEINDL, J., SYLOS-LABINI, P. et all, Progresso Técnico e Teoria Econômica. São Paulo: Hucitec/Unicamp, p.1-38.

GIBSON, B. \& MCLEUD, D. (1983) “ Non-Produced Means of Production in Sraffa’s System: Basics, Non-Basics and Quasi-Basics”. Cambridge Journal of Economics, v. 7, n.2, June, p.107-116.

HARCOURT, G. C., LANG, N.F. (1971) Capital and Growth. Middlesex: Penguin Books.

HARCOURT, G. C. (1972) Some Cambridge Controversies in the Theory of Capital. Cambridge University Press.

HOFFMANN, R.; VENTER, P. (1990) “A Renda Extensiva da Terra em um Sistema Econômico". Pesquisa e Planejamento Econômico, v.20, n.3, dezembro, p.601-612.

KURZ, H. (1980) "Rent Theory in a Multisectoral Model”. Oxford Economic Papers, v.30, n.1, p.1637.

MARX, K. (1985) O Capital: Crítica da Economia Política. 4a ed. São Paulo, DIFEL, Livro 3, v.6.

MÉDIO, A. (1973) "Profits and Surplus-Value: Appearance and Reality in Capitalist Production". In: HUNT, E.K.; SCHWARTZ, J. C. (ed). A critique of Economic Theory. Harmondsworth: Penguin Books, p. 312-346.

MONTANI, C. (1975) “Scarce Natural Resources and Income Distribution”. Metroeconomica, v.17, n. 1, jan-abr, p. 68-101.

MORISHIMA, M. (1973) Marx's Economic's: a Dual Theory of Value and Growth. Cambridge University Press, 1973, p. 198.

MORISHIMA, M. (1 974) “Marx in the Light of Modem Economic Theory”. Econometrica. v. 42, n.4, July, p. 611-632.

MORISHIMA, M. (1976) "Positive Profits with Negative Surplus Value - a Comment". The Economic Journal, v. 86, p. 599-603, September.

MORISHIMA, M.; C:ATEPHORES, G. (1980) Valor, Exploração e Crescimento: Marx à Luz da Teoria Econômica Moderna. Rio de Janeiro: Zahar Editores.

PASINETTI, L. I. (1977) Lectures on The Theory of Production. New York: Columbia University Press.

PASINETTI, L. I. (1980) Essays on The Theory of Joint Production. London: Unwin Bros. p.12-17: Introductory Note: Joint Pro duction.

QUADRIO-CURZIO, A. (1980) “Rent, Income Distribution, and Orders of Efficiency and Rentability”. In: PASINETTI, L. I. Essays on The Theory of Joint Production. New York: Columbia University Press, p.218-240.

RAVINDRAN, A., PHILLIPS, D.T., SOLBERG, J. J. (1987) Operations Research: Principles and Practice. 2.ed. New York: John Wiley.

RONCAGLIA, A. (1978) Sraffa and The Theory of Prices. New York: John Wiley.

SRAFFA, P. (1985) Produção de Mercadorias por Meio de Mercadorias: Prelúdio a uma Crítica da Teoria Econômica. 2.ed. São Paulo: Abril Cultural, p. 172- 258. (Os Economistas) 
STEEDMAN, 1. (1975) "Positive Profits with Negative Surplus Value". The Economic Journal. v.85, n..137, p. 1 14-121, March.

STEEDMAN, I. (1976) "Positive Profits with Negative Surplus Value: a Reply". The Economic Journal, v.86, p.604-608, September.

STEEDMAN, I. (1977) Marx after Sraffa. London: Unwin Brothers Limited.

SWANSON, P. A. (1986) “The Labor Theory of Value and Fixed Capital". Review of Radical Political Economics. v.18, n.3, p.44-64.

WOODS, J. E. (1990) The Production of Commodities: an Introduction to Sraffa. Basingstone: The MacMillan Press, 1 990, p. 344. 